Kragujevac Journal of Mathematics

Volume 42(1) (2018), Pages 69-82.

\title{
PERIODIC SOLUTIONS FOR IMPULSIVE NEUTRAL DYNAMIC EQUATIONS WITH INFINITE DELAY ON TIME SCALES
}

\author{
A. ARDJOUNI ${ }^{1}$ AND A. DJOUDI ${ }^{2}$
}

Abstract. Let $\mathbb{T}$ be a periodic time scale. We use the Krasnoselskii's fixed point theorem to show that the impulsive neutral dynamic equations with infinite delay $x^{\Delta}(t)=-A(t) x^{\sigma}(t)+g^{\Delta}(t, x(t-h(t)))+\int_{-\infty}^{t} D(t, u) f(x(u)) \Delta u, \quad t \neq t_{j}, \quad t \in \mathbb{T}$, $x\left(t_{j}^{+}\right)=x\left(t_{j}^{-}\right)+I_{j}\left(x\left(t_{j}\right)\right), \quad j \in \mathbb{Z}^{+}$

have a periodic solution. Under a slightly more stringent conditions we show that the periodic solution is unique using the contraction mapping principle.

\section{INTRODUCTION}

In 1988, Stephan Hilger [9] introduced the theory of time scales (measure chains) as a means of unifying discrete and continuum calculi. Since Hilger's initial work there has been significant growth in the theory of dynamic equations on time scales, covering a variety of different problems; see $[7,8,17]$ and references therein. The study of impulsive initial and boundary value problems is extensive. For the theory and classical results, we direct the reader to the monographs $[6,16,18]$.

Recently Althubiti, Makhzoum and Raffoul [2] investigated the existence and uniqueness of periodic solutions for the neutral differential equation with infinite delay

$$
x^{\prime}(t)=-a(t) x(t)+\frac{d}{d t} g(t, x(t-h(t)))+\int_{-\infty}^{t} D(t, u) f(x(u)) d u .
$$

By employing the Krasnoselskii's fixed point theorem and the contraction mapping principle, the authors obtained existence and uniqueness results for periodic solutions.

\footnotetext{
Key words and phrases. Periodic solutions, neutral dynamic equations, impulses, Krasnoselskii fixed point, infinite delay, time scales.

2010 Mathematics Subject Classification. Primary: 34N05, 34K13. Secondary: 34K40, 34K45.

Received: October 13, 2016.

Accepted: January 13, 2017.
} 
The nonlinear impulsive dynamic equation

$$
\begin{aligned}
& x^{\Delta}(t)=-a(t) x^{\sigma}(t)+f(t, x(t)), \quad t \neq t_{j}, t \in \mathbb{T}, \\
& x\left(t_{j}^{+}\right)=x\left(t_{j}^{-}\right)+I_{j}\left(t_{j}, x\left(t_{j}\right)\right), \quad j=1,2, \ldots, n,
\end{aligned}
$$

has been investigated in [10]. By using Schaeffer's theorem, the existence of periodic solutions has been established.

In this article, we are interested in the analysis of qualitative theory of periodic solutions of impulsive neutral dynamic equations. Inspired and motivated by the works mentioned above and the papers $[1-5,10-15,20-22]$ and the references therein, we are concerned with the system

$$
\begin{aligned}
x^{\Delta}(t)= & -A(t) x^{\sigma}(t)+g^{\Delta}(t, x(t-h(t))) \\
& +\int_{-\infty}^{t} D(t, u) f(x(u)) \triangle u, \quad t \neq t_{j}, t \in \mathbb{T}, \\
x\left(t_{j}^{+}\right)= & x\left(t_{j}^{-}\right)+I_{j}\left(x\left(t_{j}\right)\right), j \in \mathbb{Z}^{+},
\end{aligned}
$$

where $\mathbb{T}$ is an $\omega$-periodic time scale, $0 \in \mathbb{T}$ and $x^{\sigma}=x \circ \sigma$. For each interval $U$ of $\mathbb{R}$, we denote by $U_{\mathbb{T}}=U \cap \mathbb{T}, x\left(t_{j}^{+}\right)$and $x\left(t_{j}^{-}\right)$represent the right and the left limit of $x\left(t_{j}\right)$ in the sense of time scales, in addition, if $t_{j}$ is left-scattered, then $x\left(t_{j}^{-}\right)=x\left(t_{j}\right), A(t)=$ $\operatorname{diag}\left(a_{i}(t)\right)_{n \times n}\left(a_{i} \in \mathcal{R}^{+}\right)$and $D(t, u)=\operatorname{diag}\left(D_{i}(t, u)\right)_{n \times n}\left(D_{i} \in C(\mathbb{T}, \mathbb{R})\right)$ are diagonal matrices with continuous real-valued functions as its elements, $\mathcal{R}^{+}=\{a \in C(\mathbb{T}, \mathbb{R}): 1+$ $\mu(t) a(t)>0\}$ where $\mu(t)=\sigma(t)-t, h \in C(\mathbb{T}, \mathbb{T}), g=\left(g_{1}, g_{2}, \ldots, g_{n}\right) \in C\left(\mathbb{T} \times \mathbb{R}^{n}, \mathbb{R}^{n}\right)$, $f=\left(f_{1}, f_{2}, \ldots, f_{n}\right) \in C\left(\mathbb{R}^{n}, \mathbb{R}^{n}\right), I_{j}=\left(I_{j}^{(1)}, I_{j}^{(2)}, \ldots, I_{j}^{(n)}\right) \in C\left(\mathbb{R}^{n}, \mathbb{R}^{n}\right)$ and $A(t), h(t)$, $g(t, x(t-h(t)))$ are all $\omega$-periodic functions with respect to $t, D(t+\omega, u+\omega)=$ $D(t, u), \omega>0$ is a constant. There exists a positive integer $p$ such that $t_{j+p}=t_{j}+\omega$, $I_{j+p}=I_{j}, j \in \mathbb{Z}^{+}$, without loss of generality, we also assume that $[0, \omega)_{\mathbb{T}} \cap\left\{t_{j}, j \in\right.$ $\left.\mathbb{Z}^{+}\right\}=\left\{t_{1}, t_{2}, \ldots, t_{p}\right\}$.

To reach our desired end we have to transform the system (1.1) into an integral system and then use Krasnoselskii's fixed point theorem to show the existence of periodic solutions. The obtained integral system is the sum of two mappings, one is a contraction and the other is a compact. Also, transforming system (1.1) to an integral system enables us to show the uniqueness of the periodic solution by appealing to the contraction mapping principle.

The organization of this paper is as follows. In Section 2, we introduce some notations and definitions, and state some preliminary results needed in later sections, then we give the Green's function of (1.1), which plays an important role in this paper. In Section 3, we establish our main results for periodic solutions by applying the Krasnoselskii's fixed point theorem and the contraction mapping principle.

\section{Preliminaries}

In this section, we shall recall some basic definitions and lemmas which are used in what follows. 
Let $\mathbb{T}$ be a nonempty closed subset (time scale) of $\mathbb{R}$. The forward and backward jump operators $\sigma, \rho: \mathbb{T} \rightarrow \mathbb{T}$ and the graininess $\mu: \mathbb{T} \rightarrow \mathbb{R}^{+}$are defined, respectively, by

$$
\sigma(t)=\inf \{s \in \mathbb{T}: s>t\}, \quad \rho(t)=\sup \{s \in \mathbb{T}: s<t\}, \quad \mu(t)=\sigma(t)-t .
$$

A point $t \in \mathbb{T}$ is called left-dense if $t>\inf \mathbb{T}$ and $\rho(t)=t$, left-scattered if $\rho(t)<t$, right-dense if $t<\sup \mathbb{T}$ and $\sigma(t)=t$, and right-scattered if $\sigma(t)>t$. If $\mathbb{T}$ has a left-scattered maximum $m$, then $\mathbb{T}^{k}=\mathbb{T} \backslash\{m\}$; otherwise $\mathbb{T}^{k}=\mathbb{T}$.

A function $f: \mathbb{T} \rightarrow \mathbb{R}$ is right-dense continuous (rd-continuous) provided it is continuous at right-dense point in $\mathbb{T}$ and its left-side limits exist at left-dense points in $\mathbb{T}$. If $f$ is continuous at each right-dense points and each left-dense point, then $f$ is said to be a continuous function on $\mathbb{T}$. The set of continuous functions $f: \mathbb{T} \rightarrow \mathbb{R}$ will be denoted by $C(\mathbb{T})$.

For $x: \mathbb{T} \rightarrow \mathbb{R}$ and $t \in \mathbb{T}^{k}$, we define the delta derivative of $x(t), x^{\Delta}(t)$, to be the number (if it exists) with the property that for a given $\varepsilon>0$, there exists a neighborhood $U_{\mathbb{T}}$ of $t$ such that

$$
\left|[x(\sigma(t))-x(s)]-x^{\Delta}(t)[\sigma(t)-s]\right|<\varepsilon|\sigma(t)-s|,
$$

for all $s \in U_{\mathbb{T}}$.

If $x$ is continuous, then $x$ is right-dense continuous, and if $x$ is delta differentiable at $t$, then $x$ is continuous at $t$.

Remark 2.1. $x: \mathbb{T} \rightarrow \mathbb{R}^{n}$ is delta derivable or right-dense continuous or continuous if each entry of $x$ is delta derivable or right-dense continuous or continuous.

Let $x$ be right-dense continuous. If $X^{\Delta}(t)=x(t)$, then we define the delta integral by

$$
\int_{a}^{t} x(s) \Delta s=X(t)-X(a)
$$

Definition 2.1 ([12]). We say that a time scale $\mathbb{T}$ is periodic if there exists $p>0$ such that if $t \in \mathbb{T}$, then $t \pm p \in \mathbb{T}$. For $\mathbb{T} \neq \mathbb{R}$, the smallest positive $p$ is called the period of the time scale.

Let $\mathbb{T} \neq \mathbb{R}$ be a periodic time scale with period $p$. We say that the function $f: \mathbb{T} \rightarrow \mathbb{R}$ is periodic with period $\omega$ if there exists a natural number $n$ such that $\omega=n p, f(t+\omega)=f(t)$ for all $t \in \mathbb{T}$ and $\omega$ is the smallest positive number such that $f(t+\omega)=f(t)$.

If $\mathbb{T}=\mathbb{R}$, we say that $f$ is periodic with period $\omega>0$ if $\omega$ is the smallest positive number such that $f(t+\omega)=f(t)$ for all $t \in \mathbb{T}$.

Remark 2.2. According to [12], if $\mathbb{T}$ is a periodic time scale with period $p$, then $\sigma(t+n p)=\sigma(t)+n p$ and the graininess function $\mu$ is a periodic function with period $p$. 
Definition $2.2([8])$. An $n \times n$-matrix-valued function $A$ on time scale $\mathbb{T}$ is called regressive (respect to $\mathbb{T}$ ) provided

$$
I+\mu(t) A(t)
$$

is invertible for all $t \in \mathbb{T}^{k}$.

Let $A, B: \mathbb{T} \rightarrow R^{n \times n}$ be two $n \times n$-matrix-valued regressive functions on $T$, we define

$$
\begin{aligned}
(A \oplus B)(t) & :=A(t)+B(t)+\mu(t) A(t) B(t), \\
(\ominus A)(t) & :=-[I+\mu(t) A(t)]^{-1} A(t)=-A(t)[I+\mu(t) A(t)]^{-1}, \\
(A(t)) \ominus(B(t)) & :=(A(t)) \oplus(\ominus(B(t))),
\end{aligned}
$$

for all $t \in \mathbb{T}^{k}$.

Theorem 2.1 ([8]). Let $A$ be an regressive and $r d$-continuous $n \times n$-matrix-valued function on $\mathbb{T}$ and suppose that $f: \mathbb{T} \rightarrow \mathbb{R}^{n}$ is rd-continuous. Let $t_{0} \in \mathbb{T}$ and $y_{0} \in \mathbb{R}^{n}$. Then the initial value problem

$$
y^{\Delta}=A(t) y+f(t), \quad y\left(t_{0}\right)=y_{0}
$$

has a unique solution $y: \mathbb{T} \rightarrow \mathbb{R}^{n}$.

Definition 2.3 ([8]). Let $t_{0} \in \mathbb{T}$ and assume that $A$ is an regressive and rd-continuous $n \times n$-matrix-valued function. The unique matrix-valued solution of the initial value problem

$$
x^{\Delta}(t)=A(t) x(t), \quad x\left(t_{0}\right)=I,
$$

where $I$ denotes as usual the $n \times n$-identity matrix, is called the matrix exponential function (at $\left.t_{0}\right)$, and it is denoted by $e_{A}\left(\cdot, t_{0}\right)$.

Remark 2.3. Assume that $A$ is a constant $n \times n$-matrix. If $\mathbb{T}=\mathbb{R}$, then

$$
e_{A}\left(t, t_{0}\right)=e^{A\left(t-t_{0}\right)},
$$

while if $\mathbb{T}=\mathbb{Z}$ and $I+A$ is invertible, then

$$
e_{A}\left(t, t_{0}\right)=(I+A)^{t-t_{0}} .
$$

In the following lemma, we give some properties of the matrix exponential function.

Lemma 2.1 ([8]). Assume that $A, B: \mathbb{T} \rightarrow \mathbb{R}^{n \times n}$ are regressive and $r d$-continuous matrix-valued functions on $\mathbb{T}$. Then

(i) $e_{0}(t, s) \equiv I$ and $e_{A}(t, t) \equiv I$;

(ii) $e_{A}(\sigma(t), s)=(I+\mu(t) A(t)) e_{A}(t, s)$;

(iii) $e_{A}^{-1}(t, s)=e_{\ominus A^{*}}^{*}(t, s)$;

(iv) $e_{A}(t, s)=e_{A}^{-1}(s, t)=e_{\ominus A^{*}}^{*}(s, t)$;

(v) $e_{A}(t, s) e_{A}(s, r)=e_{A}(t, r)$;

(vi) $e_{A}(t, s) e_{B}(t, s)=e_{A \oplus B}(t, s)$, if $A(t)$ and $B(t)$ commute,

where $A^{*}$ denotes the conjugate transpose of $A$. 
Lemma 2.2 ([8]). Suppose $A$ and $B$ are regressive matrix-valued functions, then

(i) $A^{*}$ is regressive;

(ii) $\ominus A^{*}=(\ominus A)^{*}$;

(iii) $\left(A^{*}\right)^{\Delta}=\left(A^{\Delta}\right)^{*}$ holds for any differential matrix-valued function $A$.

Next, we state Krasnoselskii's fixed point theorem which enables us to prove the existence of a periodic solution of (1.1). For its proof we refer the reader to [19].

Theorem 2.2 (Krasnoselskii). Let $M$ be a closed convex nonempty subset of Banach space $(B,\|\cdot\|)$. Suppose that $\Phi$ and $\Psi$ map $M$ into $B$ such that

(i) $x, y \in M$ imply $\Phi x+\Psi y \in M$;

(ii) $\Psi$ is compact and continuous;

(iii) $\Phi$ is a contraction mapping.

Then there exists $z \in M$ with $z=\Phi z+\Psi z$.

Lemma 2.3. A function $x$ is an w-periodic solution of (1.1) if and only if $x$ is an $\omega$-periodic solution of the equation

$$
\begin{aligned}
x(t)= & g(t, x(t-h(t)))+\int_{t}^{t+\omega} G(t, s)\left[\int_{-\infty}^{s} D(s, u) f(x(u)) \triangle u\right. \\
& \left.-A(s) g^{\sigma}(s, x(s-h(s)))\right] \Delta s+\sum_{j: t_{j} \in[t, t+\omega)} G\left(t, t_{j}\right) I_{j}\left(x\left(t_{j}\right)\right),
\end{aligned}
$$

where

$$
\begin{aligned}
G(t, s) & =\operatorname{diag}\left(G_{i}(t, s)\right)_{n \times n}, G_{i}(t, s)=\left(1-e_{\ominus a_{i}}(\omega, 0)\right)^{-1} e_{\ominus a_{i}}(t+\omega, s), \\
A(t) & =\operatorname{diag}\left(a_{i}(t)\right)_{n \times n}, e_{\ominus a_{i}}(t, s)=\frac{1}{e_{a_{i}}(t, s)} \\
\ominus a_{i}(t) & =-\frac{a_{i}(t)}{1+\mu(t) a_{i}(t)}, g^{\sigma}(t, x(t-h(t)))=g\left(\sigma(t), x^{\sigma}(t-h(t))\right) .
\end{aligned}
$$

Proof. If $x$ is an $\omega$-periodic solution of (1.1). For any $t \in \mathbb{T}$, there exists $j \in \mathbb{Z}$ such that $t_{j}$ is the first impulsive point after $t$. Then for $i=1,2, \ldots, n, x_{i}$ is an $\omega$-periodic solution of the equation

$$
x_{i}^{\Delta}(t)+a_{i}(t) x_{i}^{\sigma}(t)=g_{i}^{\Delta}\left(t, x_{i}(t-h(t))\right)+\int_{-\infty}^{t} D_{i}(t, u) f_{i}(x(u)) \triangle u .
$$

Multiply both sides of $(2.1)$ by $e_{a_{i}}(t, 0)$ and then integrate from $t$ to $s \in\left[t, t_{j}\right]_{\mathbb{T}}$, we obtain

$$
\begin{aligned}
& \int_{t}^{s}\left[e_{a_{i}}(\tau, 0) x_{i}(\tau)\right]^{\Delta} \Delta \tau \\
= & \int_{t}^{s} e_{a_{i}}(\tau, 0)\left[g_{i}^{\Delta}\left(\tau, x_{i}(\tau-h(\tau))\right)+\int_{-\infty}^{\tau} D_{i}(\tau, u) f_{i}\left(x_{i}(u)\right) \Delta u\right] \Delta \tau
\end{aligned}
$$


or

$$
\begin{aligned}
e_{a_{i}}(s, 0) x_{i}(s)= & e_{a_{i}}(t, 0) x_{i}(t)+\int_{t}^{s} e_{a_{i}}(\tau, 0)\left[g_{i}^{\Delta}\left(\tau, x_{i}(\tau-h(\tau))\right)\right. \\
& \left.+\int_{-\infty}^{\tau} D_{i}(\tau, u) f_{i}\left(x_{i}(u)\right) \triangle u\right] \Delta \tau,
\end{aligned}
$$

then

$$
\begin{aligned}
x_{i}(s)= & e_{\ominus a_{i}}(s, t) x_{i}(t)+\int_{t}^{s} e_{\ominus a_{i}}(s, \tau)\left[g_{i}^{\Delta}\left(\tau, x_{i}(\tau-h(\tau))\right)\right. \\
& \left.+\int_{-\infty}^{\tau} D_{i}(\tau, u) f_{i}\left(x_{i}(u)\right) \triangle u\right] \Delta \tau, \quad i=1,2, \ldots, n,
\end{aligned}
$$

hence

$$
\begin{aligned}
x_{i}\left(t_{j}\right)= & e_{\ominus a_{i}}\left(t_{j}, t\right) x_{i}(t)+\int_{t}^{t_{j}} e_{\ominus a_{i}}\left(t_{j}, \tau\right)\left[g_{i}^{\Delta}\left(\tau, x_{i}(\tau-h(\tau))\right)\right. \\
& \left.+\int_{-\infty}^{\tau} D_{i}(\tau, u) f_{i}\left(x_{i}(u)\right) \triangle u\right] \Delta \tau, \quad i=1,2, \ldots, n .
\end{aligned}
$$

Similarly, for $s \in\left(t_{j}, t_{j+1}\right]$, we have

$$
\begin{aligned}
x_{i}(s)= & e_{\ominus a_{i}}\left(s, t_{j}\right) x_{i}\left(t_{j}^{+}\right)+\int_{t_{j}}^{s} e_{\ominus a_{i}}(s, \tau)\left[g_{i}^{\Delta}\left(\tau, x_{i}(\tau-h(\tau))\right)\right. \\
& \left.+\int_{-\infty}^{\tau} D_{i}(\tau, u) f_{i}\left(x_{i}(u)\right) \Delta u\right] \Delta \tau \\
= & e_{\ominus a_{i}}\left(s, t_{j}\right) x_{i}\left(t_{j}^{-}\right)+\int_{t_{j}}^{s} e_{\ominus a_{i}}(s, \tau)\left[g_{i}^{\Delta}\left(\tau, x_{i}(\tau-h(\tau))\right)\right. \\
& \left.+\int_{-\infty}^{\tau} D_{i}(\tau, u) f_{i}\left(x_{i}(u)\right) \Delta u\right] \Delta \tau+e_{\ominus a_{i}}\left(s, t_{j}\right) I_{j}^{(i)}\left(x_{i}\left(t_{j}\right)\right) \\
= & e_{\ominus a_{i}}\left(s, t_{j}\right) x_{i}\left(t_{j}\right)+\int_{t_{j}}^{s} e_{\ominus a_{i}}(s, \tau)\left[g_{i}^{\Delta}\left(\tau, x_{i}(\tau-h(\tau))\right)\right. \\
& \left.+\int_{-\infty}^{\tau} D_{i}(\tau, u) f_{i}\left(x_{i}(u)\right) \triangle u\right] \Delta \tau+e_{\ominus a_{i}}\left(s, t_{j}\right) I_{j}^{(i)}\left(x_{i}\left(t_{j}\right)\right),
\end{aligned}
$$

for $i=1,2, \ldots, n$. Substituting $(2.2)$ in the above equality, we obtain

$$
\begin{aligned}
x_{i}(s)= & e_{\ominus a_{i}}(s, t) x_{i}(t)+\int_{t}^{s} e_{\ominus a_{i}}(s, \tau)\left[g_{i}^{\Delta}\left(\tau, x_{i}(\tau-h(\tau))\right)\right. \\
& \left.+\int_{-\infty}^{\tau} D_{i}(\tau, u) f_{i}\left(x_{i}(u)\right) \triangle u\right] \Delta \tau+e_{\ominus a_{i}}\left(s, t_{j}\right) I_{j}^{(i)}\left(x_{i}\left(t_{j}\right)\right) .
\end{aligned}
$$

Repeating the above process for $s \in[t, t+\omega]_{\mathbb{T}}$, we have

$$
x_{i}(s)=e_{\ominus a_{i}}(s, t) x_{i}(t)+\int_{t}^{s} e_{\ominus a_{i}}(s, \tau)\left[g_{i}^{\Delta}\left(\tau, x_{i}(\tau-h(\tau))\right)\right.
$$




$$
\left.+\int_{-\infty}^{\tau} D_{i}(\tau, u) f_{i}\left(x_{i}(u)\right) \Delta u\right] \Delta \tau+\sum_{j: t_{j} \in[t, t+\omega)} e_{\ominus a_{i}}\left(s, t_{j}\right) I_{j}^{(i)}\left(x_{i}\left(t_{j}\right)\right),
$$

for $i=1,2, \ldots, n$. Let $s=t+\omega$ in the above equality, we have

$$
\begin{aligned}
x_{i}(t+\omega)= & e_{\ominus a_{i}}(t+\omega, t) x_{i}(t)+\int_{t}^{t+\omega} e_{\ominus a_{i}}(t+\omega, \tau)\left[g_{i}^{\Delta}\left(\tau, x_{i}(\tau-h(\tau))\right)\right. \\
& \left.+\int_{-\infty}^{\tau} D_{i}(\tau, u) f_{i}\left(x_{i}(u)\right) \Delta u\right] \Delta \tau+\sum_{j: t_{j} \in[t, t+\omega)} e_{\ominus a_{i}}\left(t+\omega, t_{j}\right) I_{j}^{(i)}\left(x_{i}\left(t_{j}\right)\right),
\end{aligned}
$$

$i=1,2, \ldots, n$. Noticing that $x_{i}(t+\omega)=x_{i}(t)$ and $e_{\ominus a_{i}}(t+\omega, t)=e_{\ominus a_{i}}(\omega, 0)$, we obtain

$$
\begin{aligned}
\left(1-e_{\ominus a_{i}}(\omega, 0)\right) x_{i}(t)= & \int_{t}^{t+\omega} e_{\ominus a_{i}}(t+\omega, \tau)\left[g_{i}^{\Delta}\left(\tau, x_{i}(\tau-h(\tau))\right)\right. \\
& \left.+\int_{-\infty}^{\tau} D_{i}(\tau, u) f_{i}\left(x_{i}(u)\right) \triangle u\right] \Delta \tau \\
& +\sum_{j: t_{j} \in[t, t+\omega)} e_{\ominus a_{i}}\left(t+\omega, t_{j}\right) I_{j}^{(i)}\left(x_{i}\left(t_{j}\right)\right),
\end{aligned}
$$

for $i=1,2, \ldots, n$. Notice that

$$
\begin{aligned}
& \int_{t}^{t+\omega} e_{\ominus a_{i}}(t+\omega, \tau) g_{i}^{\Delta}\left(\tau, x_{i}(\tau-h(\tau))\right) \Delta \tau \\
= & e_{\ominus a_{i}}(t+\omega, t+\omega) g_{i}\left(t+\omega, x_{i}(t+\omega-h(t+\omega))\right) \\
& -e_{\ominus a_{i}}(t+\omega, t) g_{i}\left(t, x_{i}(t-h(t))\right) \\
& -\int_{t}^{t+\omega} e_{\ominus a_{i}}(t+\omega, \tau) a_{i}(\tau) g_{i}^{\sigma}\left(\tau, x_{i}(\tau-h(\tau))\right) \Delta \tau \\
= & {\left[1-e_{\ominus a_{i}}(\omega, 0)\right] g_{i}\left(t, x_{i}(t-h(t))\right) } \\
& -\int_{t}^{t+\omega} e_{\ominus a_{i}}(t+\omega, \tau) a_{i}(\tau) g_{i}^{\sigma}\left(\tau, x_{i}(\tau-h(\tau))\right) \Delta \tau, \quad i=1,2, \ldots, n .
\end{aligned}
$$

It follows from (2.3) and (2.4) that

$$
\begin{aligned}
x_{i}(t)= & g_{i}\left(t, x_{i}(t-h(t))\right)+\int_{t}^{t+\omega}\left[1-e_{\ominus a_{i}}(\omega, 0)\right]^{-1} e_{\ominus a_{i}}(t+\omega, \tau) \\
& \times\left[\int_{-\infty}^{\tau} D_{i}(\tau, u) f_{i}\left(x_{i}(u)\right) \triangle u-a_{i}(\tau) g_{i}^{\sigma}\left(\tau, x_{i}(\tau-h(\tau))\right)\right] \Delta \tau \\
& +\sum_{j: t_{j} \in[t, t+\omega)}\left[1-e_{\ominus a_{i}}(\omega, 0)\right]^{-1} e_{\ominus a_{i}}\left(t+\omega, t_{j}\right) I_{j}^{(i)}\left(x_{i}\left(t_{j}\right)\right) \\
= & g_{i}\left(t, x_{i}(t-h(t))\right)+\int_{t}^{t+\omega} G_{i}(t, \tau)\left[\int_{-\infty}^{\tau} D_{i}(\tau, u) f_{i}\left(x_{i}(u)\right) \triangle u\right.
\end{aligned}
$$




$$
\left.-a_{i}(\tau) g_{i}^{\sigma}\left(\tau, x_{i}(\tau-h(\tau))\right)\right] \Delta \tau+\sum_{j: t_{j} \in[t, t+\omega)} G_{i}\left(t, t_{j}\right) I_{j}^{(i)}\left(x_{i}\left(t_{j}\right)\right),
$$

for $i=1,2, \ldots, n$. Next, we prove the converse. Let

$$
\begin{aligned}
x_{i}(t)=g_{i}(t, & \left.x_{i}(t-h(t))\right)+\int_{t}^{t+\omega} G_{i}(t, s)\left[\int_{-\infty}^{s} D_{i}(s, u) f_{i}\left(x_{i}(u)\right) \Delta u\right. \\
& \left.-a_{i}(s) g_{i}^{\sigma}\left(s, x_{i}(s-h(s))\right)\right] \Delta s+\sum_{j: t_{j} \in[t, t+\omega)} G_{i}\left(t, t_{j}\right) I_{j}^{(i)}\left(x_{i}\left(t_{j}\right)\right),
\end{aligned}
$$

where

$$
G_{i}(t, s)=\left(1-e_{\ominus a_{i}}(\omega, 0)\right)^{-1} e_{\ominus a_{i}}(t+\omega, s), \quad i=1,2, \ldots, n .
$$

Then if $t \neq t_{i}, i \in \mathbb{Z}^{+}$, we have

$$
\begin{aligned}
& x_{i}^{\Delta}(t) \\
= & g_{i}^{\Delta}\left(t, x_{i}(t-h(t))\right) \\
& +\int_{t}^{t+\omega}\left\{G_{i}(t, s)\left[\int_{-\infty}^{s} D_{i}(s, u) f_{i}\left(x_{i}(u)\right) \Delta u-a_{i}(s) g_{i}^{\sigma}\left(s, x_{i}(s-h(s))\right)\right]\right\}^{\Delta} \Delta s \\
& +G_{i}(t, t+\omega)\left[\int_{-\infty}^{t+\omega} D_{i}(t+\omega, u) f_{i}\left(x_{i}(u)\right) \Delta u\right. \\
& \left.\quad-a_{i}(t+\omega) g_{i}^{\sigma}\left(t+\omega, x_{i}(t+\omega-h(t+\omega))\right)\right] \\
& -G_{i}(t, t)\left[\int_{-\infty}^{t} D_{i}(t, u) f_{i}\left(x_{i}(u)\right) \Delta u-a_{i}(t) g_{i}^{\sigma}\left(t, x_{i}(t-h(t))\right)\right] \\
= & g_{i}^{\Delta}\left(t, x_{i}(t-h(t))\right)+\int_{-\infty}^{t} D_{i}(t, u) f_{i}\left(x_{i}(u)\right) \Delta u-a_{i}(t) g_{i}^{\sigma}\left(t, x_{i}(t-h(t))\right) \\
& +\int_{t}^{t+\omega}\left\{G_{i}(t, s)\left[\int_{-\infty}^{s} D_{i}(s, u) f_{i}\left(x_{i}(u)\right) \triangle u-a_{i}(s) g_{i}^{\sigma}\left(s, x_{i}(s-h(s))\right)\right]\right\}^{\Delta} \Delta s \\
= & g_{i}^{\Delta}\left(t, x_{i}(t-h(t))\right)+\int_{-\infty}^{t} D_{i}(t, u) f_{i}\left(x_{i}(u)\right) \Delta u-a_{i}(t) x_{i}^{\sigma}(t) \\
= & -a_{i}(t) x_{i}^{\sigma}(t)+g_{i}^{\Delta}\left(t, x_{i}(t-h(t))\right)+\int_{-\infty}^{t} D_{i}(t, u) f_{i}\left(x_{i}(u)\right) \triangle u, \quad i=1,2, \ldots, n .
\end{aligned}
$$

If $t=t_{i}, i \in \mathbb{Z}^{+}$, we obtain

$$
\begin{aligned}
x_{i}\left(t_{i}^{+}\right)-x_{i}\left(t_{i}^{-}\right) & =\sum_{j: t_{j} \in\left[t_{i}^{+}, t_{i}^{+}+\omega\right)} G_{i}\left(t_{i}, t_{j}\right) I_{j}^{(i)}\left(x_{i}\left(t_{j}\right)\right)-\sum_{j: t_{j} \in\left[t_{i}^{-}, t_{i}^{-}+\omega\right)} G_{i}\left(t_{i}, t_{j}\right) I_{j}^{(i)}\left(x_{i}\left(t_{j}\right)\right) \\
& =G_{i}\left(t_{i}, t_{i}+\omega\right) I_{i}^{(i)}\left(x_{i}\left(t_{i}+\omega\right)\right)-G_{i}\left(t_{i}, t_{i}\right) I_{i}^{(i)}\left(x_{i}\left(t_{i}\right)\right) \\
& =I_{i}^{(i)}\left(x_{i}\left(t_{i}\right)\right), i=1,2, \ldots, n .
\end{aligned}
$$


So we know that, $x$ is also an $\omega$-periodic solution of (1.1). This completes the proof.

Throughout this paper, we make the following assumptions.

(H1) The function $g=\left(g_{1}, g_{2}, \ldots, g_{n}\right)$ satisfies a Lipschitz condition in $x$. That is, for $i \in\{1,2, \ldots, n\}$, there exists a positive constant $L_{i}$ such that

$$
\left|g_{i}(t, x)-g_{i}(t, y)\right| \leq L_{i}\|x-y\|, \quad \text { for all } t \in \mathbb{T}, x, y \in \mathbb{R}^{n} .
$$

(H2) The function $f=\left(f_{1}, f_{2}, \ldots, f_{n}\right)$ satisfies a Lipschitz condition in $x$. That is, for $i \in\{1,2, \ldots, n\}$, there exists a positive constants $M_{i}$ such that

$$
\left|f_{i}(x)-f_{i}(y)\right| \leq M_{i}\|x-y\|, \quad \text { for all } t \in \mathbb{T}, x, y \in \mathbb{R}^{n} .
$$

(H3) For $j \in \mathbb{Z}, I_{j}=\left(I_{j}^{(1)}, I_{j}^{(2)}, \ldots, I_{j}^{(n)}\right)$ satisfies Lipschitz condition. That is, for $i \in\{1,2, \ldots, n\}$ there exists a positive constant $P_{j}^{(i)}$ such that

$$
\left|I_{j}^{(i)}(x)-I_{j}^{(i)}(y)\right| \leq P_{j}^{(i)}\|x-y\|, \quad \text { for all } x, y \in \mathbb{R}^{n} .
$$

(H4) There exists a positive constant $N_{i}$ such that

$$
\int_{-\infty}^{t}\left|D_{i}(t, u)\right| \triangle u \leq N_{i}
$$

To apply Theorem 2.2 to (1.1), we define

$P C(\mathbb{T})=\left\{x: \mathbb{T} \rightarrow \mathbb{R}^{n}:\left.x\right|_{\left(t_{j}, t_{j+1}\right)_{\mathbb{T}}} \in C\left(t_{j}, t_{j+1}\right)_{\mathbb{T}}, \exists x\left(t_{j}^{-}\right)=x\left(t_{j}\right), x\left(t_{j}^{+}\right), j \in \mathbb{Z}^{+}\right\}$.

Consider the Banach space

$$
X=\{x \in P C(\mathbb{T}): x(t+\omega)=x(t)\},
$$

with the norm $\|x\|=\max _{t \in[0, \omega]_{\mathbb{T}}}|x(t)|_{0}$, where $|x(t)|_{0}=\max _{1 \leq i \leq n}\left|x_{i}(t)\right|$.

Lemma 2.4 ([12]). Let $x \in X$. Then there exists $\left\|x^{\sigma}\right\|$, and $\left\|x^{\sigma}\right\|=\|x\|$.

Noticing that

$$
G_{i}(t, s) \leq\left(1-e_{\ominus a_{i}}(\omega, 0)\right)^{-1}:=\eta_{i},
$$

for convenience, we introduce the notation

$$
\begin{aligned}
\bar{\eta} & :=\max _{1 \leq i \leq n} \eta_{i}, \quad \gamma:=\max _{1 \leq i \leq n} \max _{t \in[0, \omega]_{\mathbb{T}}}\left|a_{i}(t)\right|, \quad L:=\max _{1 \leq i \leq n} L_{i}, \quad M:=\max _{1 \leq i \leq n} M_{i}, \\
N & :=\max _{1 \leq i \leq n} N_{i}, \quad P_{j}:=\max _{1 \leq i \leq n} P_{j}^{(i)}, \quad P:=\max _{1 \leq j \leq p} P_{j} .
\end{aligned}
$$

Define the mapping $H: X \rightarrow X$ by

$$
\begin{aligned}
(H \varphi)(t)=g(t, \varphi(t-h(t)))+\int_{t}^{t+\omega} G(t, s)\left[\int_{-\infty}^{s} D(s, u) f(\varphi(u)) \Delta u\right. \\
\left.-A(s) g^{\sigma}(s, \varphi(s-h(s)))\right] \Delta s+\sum_{j: t_{j} \in[t, t+\omega)} G\left(t, t_{j}\right) I_{j}\left(x\left(t_{j}\right)\right) .
\end{aligned}
$$


To apply Theorem 2.2, we need to construct two mappings: one is a contraction and the other is continuous and compact. We express (2.5) as

$$
(H \varphi)(t)=(\Phi \varphi)(t)+(\Psi \varphi)(t)
$$

where

$$
(\Phi \varphi)(t)=g(t, \varphi(t-h(t)))
$$

and

$$
\begin{aligned}
& (\Psi \varphi)(t) \\
= & \int_{t}^{t+\omega} G(t, s)\left[\int_{-\infty}^{s} D(s, u) f(\varphi(u)) \triangle u-A(s) g^{\sigma}(s, \varphi(s-h(s)))\right] \Delta s \\
& +\sum_{j: t_{j} \in[t, t+\omega)} G\left(t, t_{j}\right) I_{j}\left(\varphi\left(t_{j}\right)\right) .
\end{aligned}
$$

Lemma 2.5. Suppose (H1) holds and $L<1$, then $\Phi: X \rightarrow X$, as defined by (2.6), is a contraction.

Proof. Trivially, $\Phi: X \rightarrow X$. For $\varphi, \psi \in X$, we have

$$
\begin{aligned}
\|\Phi(\varphi)-\Phi(\psi)\| & =\max _{t \in[0, \omega]_{\mathbb{T}}} \max _{1 \leq i \leq n}\left|g_{i}\left(t, \varphi_{i}(t-h(t))\right)-g_{i}\left(t, \psi_{i}(t-h(t))\right)\right| \\
& \leq L\|\varphi-\psi\| .
\end{aligned}
$$

Hence $\Phi$ defines a contraction mapping with contraction constant $L$.

Lemma 2.6. Suppose (H1)-(H4) hold, then $\Psi: X \rightarrow X$, as defined by (2.7), is continuous and compact.

Proof. Evaluating (2.7) at $t+\omega$ gives

$$
\begin{aligned}
& (\Psi \varphi)(t+\omega) \\
= & \int_{t+\omega}^{t+2 \omega} G(t+\omega, s)\left[\int_{-\infty}^{s} D(s, u) f(\varphi(u)) \Delta u-A(s) g^{\sigma}(s, \varphi(s-h(s)))\right] \Delta s \\
& +\sum_{j: t_{j} \in[t+\omega, t+2 \omega)} G\left(t+\omega, t_{j}\right) I_{j}\left(\varphi\left(t_{j}\right)\right) . \\
= & \int_{t}^{t+\omega} G(t+\omega, v+\omega)\left[\int_{-\infty}^{v+\omega} D(v+\omega, u) f(\varphi(u)) \Delta u\right. \\
& \left.\quad-A(v+\omega) g^{\sigma}(v+\omega, \varphi(v+\omega-h(v+\omega)))\right] \Delta v+\sum_{k: t_{k} \in[t, t+\omega)} G\left(t, t_{k}\right) I_{j}\left(\varphi\left(t_{k}\right)\right) \\
= & \int_{t}^{t+\omega} G(t, v)\left[\int_{-\infty}^{v} D(v, u) f(\varphi(u)) \Delta u \sum_{k: t_{k} \in[t, t+\omega)} G\left(t, t_{k}\right) I_{j}\left(\varphi\left(t_{k}\right)\right)\right.
\end{aligned}
$$




$$
=(\Psi \varphi)(t) \text {. }
$$

That is, $\Psi: X \rightarrow X$.

Now, we show that $\Psi$ is continuous. Let $\varphi, \psi \in X$, given $\varepsilon>0$, take

$$
\delta=\frac{\varepsilon}{\bar{\eta}[\omega(M N+L \gamma)+P]},
$$

such that for $\|\varphi-\psi\| \leq \delta$. By using the Lipschitz condition, we obtain

$$
\begin{aligned}
& \|\Psi \varphi-\Psi \psi\| \\
\leq & \max _{t \in[0, \omega]_{\mathbb{T}}}\left|\int_{t}^{t+\omega} G(t, s)\left[\int_{-\infty}^{s} D(s, u) f(\varphi(u)) \Delta u-\int_{-\infty}^{s} D(s, u) f(\psi(u)) \Delta u\right] \Delta s\right|_{0} \\
& +\max _{t \in[0, \omega]_{\mathbb{T}}}\left|\int_{t}^{t+\omega} G(t, s) A(s)\left[g^{\sigma}(s, \varphi(s-h(s)))-g^{\sigma}(s, \psi(s-h(s)))\right] \Delta s\right|_{0} \\
& +\max _{t \in[0, \omega]_{\mathbb{T}}} \sum_{j: t_{j} \in[t, t+\omega)}\left|G\left(t, t_{j}\right)\left[I_{j}\left(\varphi\left(t_{j}\right)\right)-I_{j}\left(\psi\left(t_{j}\right)\right)\right]\right|_{0} \\
\leq & \bar{\eta} \int_{0}^{\omega} \int_{-\infty}^{s}|D(s, u)[f(\varphi(u))-f(\psi(u))]|_{0} \Delta u \Delta s \\
& +\bar{\eta} \gamma \int_{0}^{\omega}\left|g^{\sigma}(s, \varphi(s-h(s)))-g^{\sigma}(s, \psi(s-h(s)))\right|_{0} \Delta s \\
& +\bar{\eta} \max _{1 \leq j \leq p}\left|I_{j}\left(\varphi\left(t_{j}\right)\right)-I_{j}\left(\psi\left(t_{j}\right)\right)\right|_{0} \\
\leq & \bar{\eta}[\omega(M N+L \gamma)+P]\|\varphi-\psi\|<\varepsilon .
\end{aligned}
$$

This proves $\Psi$ is continuous. Next, we need to show that $\Psi$ is compact. Consider the sequence of periodic functions $\left\{\varphi_{n}\right\} \subset X$ and assume that the sequence is uniformly bounded. Let $\Theta>0$ be such that $\left\|\varphi_{n}\right\| \leq \Theta$, for all $n \in N$. In view of (H1)-(H3), we arrive at

$$
\begin{aligned}
\left\|g^{\sigma}(t, x)\right\| & \leq\left\|g^{\sigma}(t, x)-g^{\sigma}(t, 0)\right\|+\left\|g^{\sigma}(t, 0)\right\| \\
& =\max _{t \in[0, \omega]_{\mathbb{T}}} \max _{1 \leq i \leq n}\left|g_{i}^{\sigma}(t, x)-g_{i}^{\sigma}(t, 0)\right|+\alpha_{g} \\
& \leq L\|x\|+\alpha_{g}, \\
\|f(x)\| & \leq\|f(x)-f(0)\|+\|f(0)\| \\
& =\max _{1 \leq i \leq n}\left|f_{i}(x)-f_{i}(0)\right|+\alpha_{f} \\
& \leq M\|x\|+\alpha_{f}, \\
\left\|I_{j}(x)\right\| & \leq\left\|I_{j}(x)-I_{j}(0)\right\|+\left\|I_{j}(0)\right\| \\
& =\max _{1 \leq i \leq n} n\left|I_{j}^{(i)}(x)-I_{j}^{(i)}(0)\right|+\alpha_{I_{j}} \\
& \leq P_{j}\|x\|+\alpha_{I_{j}}, \text { for } j \in \mathbb{Z}^{+},
\end{aligned}
$$

where $\alpha_{g}=\left\|g^{\sigma}(t, 0)\right\|, \alpha_{f}=\|f(0)\|$ and $\alpha_{I_{j}}=\left\|I_{j}(0)\right\|$. Hence, 


$$
\begin{aligned}
\leq & \max _{t \in[0, \omega]_{\mathbb{T}}} \mid \int_{t}^{t+\omega} G(t, s)\left[\int_{-\infty}^{s} D(s, u) f\left(\varphi_{n}(u)\right) \Delta u\right. \\
& \left.\quad-A(s) g^{\sigma}\left(s, \varphi_{n}(s-h(s))\right)\right]\left.\Delta s\right|_{0}+\max _{t \in[0, \omega]_{\mathbb{T}}} \sum_{j: t_{j} \in[t, t+\omega)}\left|G\left(t, t_{j}\right) I_{j}\left(\varphi_{n}\left(t_{j}\right)\right)\right|_{0} \\
\leq & \bar{\eta} \int_{0}^{\omega} \int_{-\infty}^{s}\left|D(s, u) f\left(\varphi_{n}(u)\right)\right|_{0} \Delta u \Delta s+\bar{\eta} \gamma \int_{0}^{\omega}\left|g^{\sigma}\left(s, \varphi_{n}(s-h(s))\right)\right|_{0} \Delta s \\
& +\bar{\eta} \sum_{j=1}^{p}\left|I_{j}\left(\varphi_{n}\left(t_{j}\right)\right)\right|_{0} \\
\leq & \bar{\eta} \omega N\left(M\left\|\varphi_{n}\right\|+\alpha_{f}\right)+\bar{\eta} \gamma \omega\left(L\left\|\varphi_{n}\right\|+\alpha_{g}\right)+\bar{\eta}\left(\max _{1 \leq j \leq p}\left(P_{j}\left\|\varphi_{n}\right\|+\alpha_{I_{j}}\right)\right) \\
\leq & \bar{\eta} \omega \Theta(M N+\gamma L)+\bar{\eta}\left(\omega N \alpha_{f}+\gamma \omega \alpha_{g}+P \Theta+\alpha\right):=D,
\end{aligned}
$$

where $\alpha=\max _{1 \leq j \leq p} \alpha_{I_{j}}$. Thus the sequence $\left\{\Psi \varphi_{n}\right\}$ is uniformly bounded. Now, it can be easily checked that

$$
\begin{aligned}
\left(\Psi \varphi_{n}\right)^{\Delta}(t)= & -A(t)\left(\Psi \varphi_{n}\right)^{\sigma}(t)+\int_{-\infty}^{t} D(t, u) f\left(\varphi_{n}(u)\right) \triangle u \\
& -A(t) g^{\sigma}\left(t, \varphi_{n}(t-h(t))\right) .
\end{aligned}
$$

Consequently, it follows from (2.10), (2.11), (2.12) and Lemma 2.4 that

$$
\begin{aligned}
\left|\left(\Psi \varphi_{n}\right)^{\Delta}(t)\right|_{0} \leq & \|A\|\left\|\left(\Psi \varphi_{n}\right)^{\sigma}\right\|+\left\|\int_{-\infty}^{t} D(t, u) f\left(\varphi_{n}(u)\right) \Delta u\right\| \\
& +\left|A(t) g^{\sigma}\left(t, \varphi_{n}(t-h(t))\right)\right|_{0} \\
\leq & \|A\|\left\|\left(\Psi \varphi_{n}\right)\right\|+N\left(M\left\|\varphi_{n}\right\|+\alpha_{f}\right)+\|A\|\left(L\left\|\varphi_{n}\right\|+\alpha_{g}\right) \\
\leq & \|A\|\left(D+L \Theta+\alpha_{g}\right)+N\left(M \Theta+\alpha_{f}\right),
\end{aligned}
$$

for all $n$. That is, $\left\|\left(\Psi \varphi_{n}\right)^{\Delta}\right\| \leq\|A\|\left(D+L \Theta+\alpha_{g}\right)+N\left(M \Theta+\alpha_{f}\right)$, thus the sequence $\left\{\Psi \varphi_{n}\right\}$ is uniformly bounded and equi-continuous. The Arzela-Ascoli theorem implies that $\Psi$ is compact.

\section{MAin Results}

Our main results reads as follows.

Theorem 3.1. Assume that (H1)-(H4) hold and $L<1$. Suppose that there is a positive constant $G$ such that all solutions $x$ of (1.1), $x \in X$, satisfy $\|x\| \leq G$, and the inequality

$$
\frac{\gamma \omega \alpha_{g}+\omega N \alpha_{f}+\alpha}{1 / \bar{\eta}-\omega(\gamma L+M N)-L / \bar{\eta}-P} \leq G,
$$

holds. Then (1.1) has an $\omega$-periodic solution. 
Proof. Define $M=\{\varphi \in X:\|\varphi\| \leq G\}$. Then Lemma 2.6 implies $\Psi: X \rightarrow X$ and $\Psi$ is compact and continuous. Also, from Lemma 2.5, the mapping $\Phi$ is a contraction and $\Phi: X \rightarrow X$. We need to show that if $\varphi, \psi \in M$, then $\|\Phi \varphi+\Psi \psi\| \leq G$. Let $\varphi, \psi \in M$ with $\|\varphi\|,\|\psi\| \leq G$, from (2.9)-(2.11), we have

$$
\begin{aligned}
\|\Phi \varphi+\Psi \psi\| & \leq\|\Phi \varphi\|+\|\Psi \psi\| \\
& \leq L G+\bar{\eta} \omega G(\gamma L+M N)+\bar{\eta}\left(\gamma \omega \alpha_{g}+\omega N \alpha_{f}+G P+\alpha\right) \leq G .
\end{aligned}
$$

Thus $\Phi \varphi+\Psi \psi \in M$. We see that all the conditions of Krasnoselskii theorem are satisfied on the set $M$. Hence there exists a fixed point $z$ in $M$ such that $z=\Phi z+\Psi z$. By Lemma 2.3, this fixed point is a solution of (1.1).

Theorem 3.2. Suppose that (H1)-(H4) hold. If

$$
\Upsilon:=\bar{\eta}[\omega(\gamma L+M N)+P]<1,
$$

then (1.1) has an unique $\omega$-periodic solution.

Proof. For $\varphi, \psi \in X$, we have

$$
\begin{aligned}
&\|H \varphi-H \psi\| \leq \bar{\eta} \int_{0}^{\omega} \int_{-\infty}^{s}|D(s, u) f(\varphi(u))-f(\psi(u))|_{0} \Delta u \Delta s \\
&+\bar{\eta} \gamma \int_{0}^{\omega}\left|g^{\sigma}(s, \varphi(s-h(s)))-g^{\sigma}(s, \psi(s-h(s)))\right|_{0} \Delta s \\
&+\bar{\eta} \sum_{j=1}^{p}\left|I_{j}\left(\varphi\left(t_{j}\right)\right)-I_{j}\left(\psi\left(t_{j}\right)\right)\right|_{0} \\
& \leq \bar{\eta} \omega M N\|\varphi-\psi\|+\bar{\eta} \gamma \omega L\|\varphi-\psi\|+\bar{\eta} P\|\varphi-\psi\| \\
&<\bar{\eta}[\omega(\gamma L+M N)+P]\|\varphi-\psi\| \\
&=\Upsilon\|\varphi-\psi\| .
\end{aligned}
$$

This completes the proof.

Acknowledgements. The authors gratefully acknowledge the reviewers for their helpful comments.

\section{REFERENCES}

[1] M. Adivar and Y. N. Raffoul, Existence of periodic solutions in totally nonlinear delay dynamic equations, Electron. J. Qual. Theory Differ. Equ. 2009 (2009), 1-20.

[2] S. Althubiti, H. A. Makhzoum and Y. N. Raffoul, Periodic solution and stability in nonlinear neutral system with infinite delay, Appl. Math. Sci. 7(136) (2013), 6749-6764.

[3] A. Ardjouni and A. Djoudi, Existence of periodic solutions for nonlinear neutral dynamic equations with functional delay on a time scale, Acta Univ. Palack. Olomuc. Fac. Rerum Natur. Math. 52(1) (2013), 5-19.

[4] A. Ardjouni and A. Djoudi, Existence of periodic solutions for nonlinear neutral dynamic equations with variable delay on a time scale, Commun. Nonlinear Sci. Numer. Simul. 17 (2012), 3061-3069. 
[5] A. Ardjouni and A. Djoudi, Periodic solutions in totally nonlinear dynamic equations with functional delay on a time scale, Rend. Semin. Mat. Univ. Politec. Torino 68(4) (2010), 349359.

[6] D. D. Bainov and P. S. Simeonov, Systems with Impulse Effect: Stability, Theory and Applications, Ellis Horwood Series: Mathematics and its Applications, Ellis Horwood, 1989.

[7] M. Bohner and A. Peterson, Dynamic Equations on Time Scales, An Introduction with Applications, Birkhäuser, Boston, 2001.

[8] M. Bohner and A. Peterson, Advances in Dynamic Equations on Time Scales, Birkhäuser, Boston, 2003.

[9] S. Hilger, Ein Masskettenkalkül mit Anwendung auf Zentrumsmanningfaltigkeiten, PhD thesis, Universität Würzburg, 1988.

[10] E. R. Kaufmann, Impulsive periodic boundary value problems for dynamic equations on time scale, Adv. Difference Equ. 2009 (2009), Article ID 603271, 10 pages.

[11] E. R. Kaufmann, N. Kosmatov and Y. N. Raffoul, Impulsive dynamic equations on a time scale, Electron. J. Differential Equations 2008(67) (2008), 1-9.

[12] E. R. Kaufmann and Y. N. Raffoul, Periodic solutions for a neutral nonlinear dynamical equation on a time scale, J. Math. Anal. Appl. 319 (2006), 315-325.

[13] E. R. Kaufmann and Y. N. Raffoul, Periodicity and stability in neutral nonlinear dynamic equation with functional delay on a time scale, Electron. J. Differential Equations 2007(27) (2007), 1-12.

[14] Y. K. Li and Z. W. Xing, Existence and global exponential stability of periodic solution of CNNS with impulses, Chaos Solitons Fractals 33 (2007), 1686-1693.

[15] X. Li, X, Lin, D. Jiang and X. Zhang, Existence and multiplicity of positive periodic solutions to functional differential equations with impulse effects, Nonlinear Anal. 62 (2005), 683-701.

[16] V. Lakshmikantham, D. D. Bainov and P. S. Simeonov, Theory of Impulsive Differential Equations, Series in Modern Applied Mathematics, 6, World Scientific, New Jersey, 1994.

[17] V. Lakshmikantham, S. Sivasundaram and B. Kaymarkcalan, Dynamic Systems on Measure Chains, Kluwer Academic Publishers, Dordrecht, 1996.

[18] A. M. Samoŭlenko and N. A. Perestyuk, Impulsive Differential Equations, World Scientific Seriess on Nonlinear Science, Series A: Monographs and Treatises, 14, World Scientific, New Jersey, 1995.

[19] D. R. Smart, Fixed Points Theorems, Cambridge Univ. Press, Cambridge, UK, 1980.

[20] H. Zhang and Y. K. Li, Existence of positive solutions for functional differential equations with impulse effects on time scales, Commun. Nonlinear Sci. Numer. Simul. 14 (2009), 19-26.

[21] N. Zhang, B. Dai and X. Qian, Periodic solutions for a class of higher-dimension functional differential equations with impulses, Nonlinear Anal. 68 (2008), 629-638.

[22] X. Zhang, J. Yan and A. Zhao, Existence of positive periodic solutions for an impulsive differential equation, Nonlinear Anal. 68 (2008), 3209-3216.

${ }^{1}$ Department of Mathematics And Informatics

University of SOUK Ahras

P.O. Box 1553, Souk Ahras, Algeria

E-mail address: abd_ardjouni@yahoo.fr

${ }^{2}$ Department of Mathematics

University of ANNABA

P.O. Box 12, Annaba, Algeria

E-mail address: adjoudi@yahoo.com 